Б.Я. АЛЕКСЕЕВ, Д.М.Н., профессор, А.С. КАЛПИНСКИЙ, к.М.Н., Московский научно-исследовательский онкологический институт им П.А. Герцена, кафедра урологии с курсом онкоурологии факультета повышения квалификации медицинских работников РУДН, Москва

\title{
СОРАФЕНИБ \\ В ПОСЛЕДОВАТЕЛЬНОЙ ТЕРАПИИ \\ МЕТАСТАТИЧЕСКОГО РАКА ПОЧКИ
}

\section{С открытием патогенетических путей развития метастатического почечно-клеточного рака (МПКР) и появлением таргетных препаратов прогноз течения заболевания и выживаемость этой группы больных значительно улучшились. Резистентность и непереносимость препаратов, развивающиеся при длительной терапии, индуцируют проведение новых клинических исследований и поиск новых препаратов и схем лечения. В статье обсуждаются результаты исследований эффективности и переносимости сорафениба как в первой, так и во второй линиях лечения МПКР.}

Ключевые слова: метастатический почечно-клеточный рак, таргетная терапия, ингибиторы тирозинкиназ, сорафениб, Нексавар

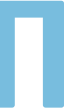

очечно-клеточный рак (ПКР) - одно из наиболее распространенных онкоурологических заболеваний.

Ежегодно в мире выявляют около 210 тыс. новых случаев ПКР (из них две трети в развитых странах), что составляет около 2\% в структуре онкологической заболеваемости [1].

В России в 2011 г. выявлено 19657 больных со злокачественными новообразованиями почки. ПКР составляет 4,5\% злокачественных новообразований у мужчин и $3 \%$ у женщин. По темпам прироста онкологической заболеваемости за период с 2001 по 2011 г. ПКР устойчиво занимает одно из ведущих мест (31,81\%). Стандартизованный показатель заболеваемости населения России злокачественными опухолями почки составил 9,06 на 100 тыс. населения. Рост заболеваемости ПКР обусловлен как истинными причинами, так и улучшением ранней

Рисунок 1. Механизм действия таргетных препаратов [7]

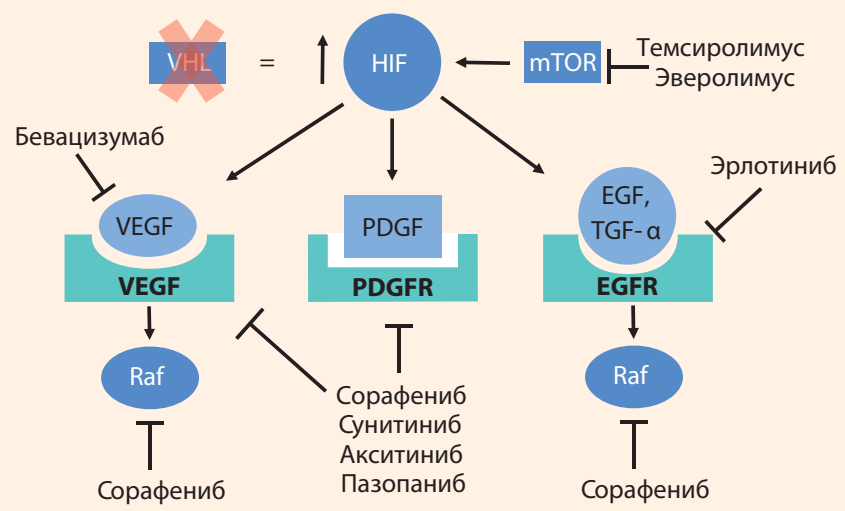

Сокращения: HIF - Hypoxia-Inducible Factor; фактор, индуцируемый гипоксией, VEGF - Vascular Endothelial Growth Factor, сосудистый эндотелиальный фактор роста; mTOR - mammalian target of rapamicin, мишень рапамицина млекопитающих; PDGF - Platelet-derived growth factor, Тромбоцитарный фактор роста; EGF - Epidermal growth factor, Эпидермальный фактор роста; VHL von Hippel-Lindau tumor suppressor, ген-супрессор опухолевого роста фон Хиппеля - Линдау, Raf Rapidly accelerated fibrosarcoma kinase, Киназа, индуцирующая быстрое развитие фибросаркомы диагностики. В настоящее время в 25-40\% случаев заболевание выявляют случайно при профилактическом обследовании [2].

Несмотря на совершенствование методов диагностики данной патологии и высокую частоту выявления (40-60\%) локализованного ПКР у 25-30\% больных ПКР при первичном обследовании обнаруживают отдаленные метастазы. у 20-40\% больных после радикально выполненного хирургического вмешательства диагностируют прогрессирование заболевания с появлением метастазов. Таким образом, распространенность запущенных форм и смертность от ПКР в мире и в России остаются высокими.

Открытие основных патогенетических путей развития ПКР, опосредованных через фактор, индуцируемый гипоксией HIF (HIF - Hypoxia-Inducible Factor), и сосудистый эндотелиальный фактор роста VEGF (VEGF - Vascular Endothelial Growth Factor), а также через мишень рапамицина млекопитающих mTOR (mTOR - mammalian target of rapamicin, мишень рапамицина млекопитающих), послужили импульсом к развитию нового лечебного направления в онкоурологии - таргетной терапии (рис. 1) [3-7].

В клинических исследованиях II и III фазы, посвященных изучению эффективности и переносимости таргетных препаратов, зарегистрировано увеличение показателей выживаемости без прогрессирования при умеренно выраженной токсичности и в некоторых исследованиях - увеличение показателей общей выживаемости больных МПКР. В настоящее время в арсенале онкологов и урологов имеются следующие таргетные препараты: сорафениб (Нексавар), сунитиниб (Сутент), темсиролимус (Торизел), бевацизумаб (Авастин), эверолимус (Афинитор), пазопаниб (Вотриент), акситиниб (Инлита). В литературе активно дискутируется вопрос о последовательном применении таргетных препаратов и выработке оптимальных режимов их применения для улучшения показателей выживаемости и качества жизни [8-10].

Существование многочисленных патогенетических путей развития ПКР обусловливает возможность последовательного применения таргетных препаратов, что позволяет полу- 
чать эффект от таргетной терапии в случаях появления резистентности к другим антиангиогенным препаратам. Последовательное использование таргетных препаратов позволяет длительно лечить больных без прогрессирования и обеспечивает возможность применения оптимальных дозировок препарата и хорошую переносимость лечения.

Клинический потенциал стратегии последовательного применения таргетных препаратов у пациентов с МПКР подтвержден рядом ретро- и проспективных клинических исследований (табл. 1, рис. 2), результаты которых указывают на отсутствие перекрестной резистентности между таргетными препаратами [6-38].

Наибольший опыт применения накоплен в отношении первых зарегистрированных и наиболее исследованных таргетных препаратов - сорафениба и сунитиниба, зарегистрированных FDA (FDA - Food and Drug Administration) в декабре 2005 г. и январе 2006 г. соответственно. Более 2 лет сорафениб и сунитиниб были единственными таргетными препаратами для лечения МПКР, в связи с чем проводилось изучение оптимальной последовательности их применения.

Сорафениб (Нексавар) относится к группе мультикиназных ингибиторов, действующих одновременно как на тирозинкиназы рецепторов (VEGFR-2, VEGFR-3, PDGF-b, RET, c-KIT, FLT-3), так и на серин/треониновые киназы (C-Raf, B-Raf) в опухолевых и эндотелиальных клетках.

Препарат сорафениб одобрили для терапии МПКР на основании данных регистрационного исследования III фазы TARGET (TARGET - Treatment Approaches in Renal Cancer Global Evaluation Trial), в которое включили 903 больных МПКР после прогрессирования на фоне лечения цитокинами (большинство больных), гормональной или химиотерапии в первой линии. Больных рандомизировали в группы лечения сорафенибом или плацебо. Риск прогрессирования или смерти в

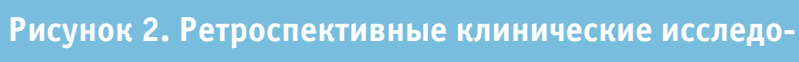

вания с использованием последовательного назначения таргетных препаратов [25-38]

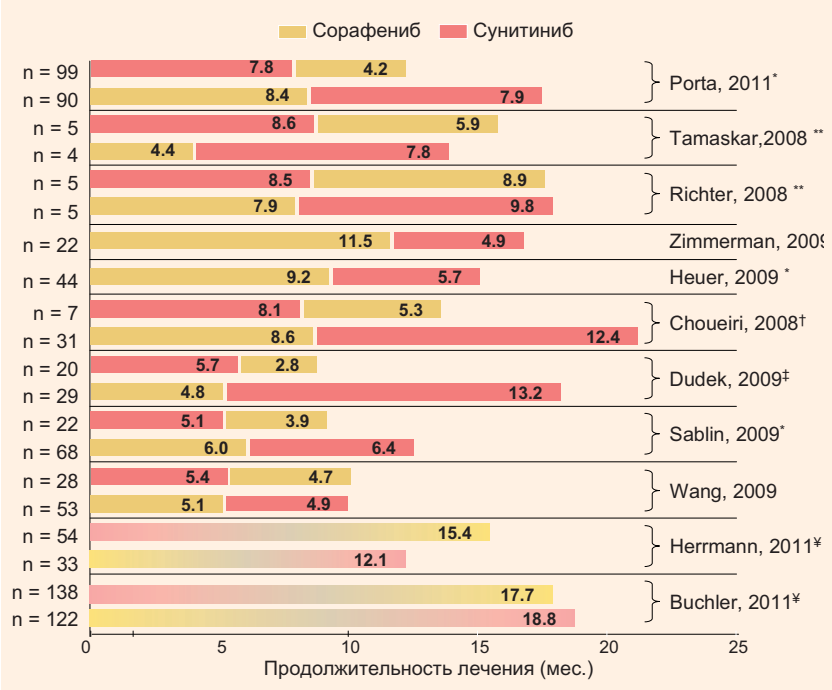

* Медиана выживаемости без прогрессирования; ** Средняя выживаемость без прогрессирования; † Продолжительность лечения; ‡ Время до прогрессирования;

¥ Общая выживаемость без прогрессирования.

группе больных, получавших сорафениб, оказался на 56\% ниже, чем в группе плацебо (отношение рисков 0,44; $95 \%$ Ди 0,35-0,55; уровень статистической значимости $\mathrm{p}=0,000001$, медиана выживаемости без прогрессирования 5,5 мес. против 2,8 мес., $\mathrm{p}<0,01)$. Частичный ответ по критериям RECIST 1.0 зарегистрировали у 10\% пациентов, получивших сорафениб, по сравнению с 2\% в группе плацебо ( $\mathrm{p}<0,001)$. Окончательный анализ общей выживаемости после исключения пациентов из группы плацебо, получивших в последующем сорафениб,

\begin{tabular}{|c|c|c|c|c|c|c|}
\hline Препарат & $\begin{array}{c}\text { Фаза } \\
\text { исследования }\end{array}$ & $\begin{array}{l}\text { Популяция } \\
\text { больных }\end{array}$ & $\begin{array}{c}\text { Количество } \\
\text { больных }\end{array}$ & $00, \%$ & Медиана ВБП & Медиана OB \\
\hline $\begin{array}{l}\text { Эверолимус } \\
\text { Плацебо [11-13] }\end{array}$ & III & $\begin{array}{l}\text { сунитиниб и/или } \\
\text { сорафениб } \\
\text { рефрактерные }\end{array}$ & 410 & 1 & $\begin{array}{l}4,9 \text { мес. } \\
1,9 \text { мес. }\end{array}$ & $\begin{array}{l}14,78 \text { мес. } \\
14,39 \text { мес. }\end{array}$ \\
\hline Сорафениб [14] & II & $\begin{array}{l}\text { сунитиниб или } \\
\text { бевацизумаб }\end{array}$ & 37 & 0 & 3,8 мес. & - \\
\hline Сорафениб [15] & II & сунитиниб & 52 & 9,6 & 16 нед. & 32 нед. \\
\hline Сунитиниб [16] & II & бевацизумаб & 61 & 23 & 30,4 нед. & 47,1 нед. \\
\hline Акситиниб [17] & II & $\begin{array}{l}\text { сунитиниб и сорафениб } \\
\text { цитокины и сорафениб } \\
\text { сорафениб }\end{array}$ & 62 & $\begin{array}{c}7,1 \\
27,6 \\
25 \\
\end{array}$ & $\begin{array}{l}\text { 7,1 мес. } \\
9,1 \text { мес. } \\
\text { 7,8 мес. }\end{array}$ & $\begin{array}{c}\text { 11,5 мес. } \\
\text { 18,5 мес. } \\
\text { 9,2 мес. }\end{array}$ \\
\hline Акситиниб [18] & II & сорафениб & 62 & 22,6 & 7,4 мес. & 13,6 мес. \\
\hline $\begin{array}{l}\text { Акситиниб } \\
\text { Сорафениб [19-20] }\end{array}$ & III & сунитиниб & $\begin{array}{l}194 \\
195\end{array}$ & $\begin{array}{c}11,3 \\
7,7 \\
\end{array}$ & $\begin{array}{l}\text { 4,8 мес. } \\
3,4 \text { мес. }\end{array}$ & $\begin{array}{l}15,2 \text { мес. } \\
16,5 \text { мес. }\end{array}$ \\
\hline $\begin{array}{l}\text { Темсиролимус } \\
\text { Сорафениб [21] }\end{array}$ & III & сунитиниб & $\begin{array}{l}259 \\
253\end{array}$ & $\begin{array}{l}7.7 \\
7.9\end{array}$ & $\begin{array}{l}\text { 4,3 мес. } \\
3,9 \text { мес. }\end{array}$ & $\begin{array}{l}12,3 \text { мес. } \\
16,6 \text { мес. }\end{array}$ \\
\hline Тивозаниб [22-24] & III & сорафениб & 156 & 13 & 8,4 мес. & 19,6 мес. \\
\hline
\end{tabular}


также выявил статистически значимое преимущество в показателях общей выживаемости в группе сорафениба по сравнению с группой плацебо: отношение рисков 0,78; 95\% Ди 0,62-0,97; $p=0,0287$, медиана общей выживаемости 17,8 мес. и 14,3 мес. соответственно ( $p=0,0287)$ [39-41].

Медиана выживаемости без прогрессирования в исследованиях II и III фазы сорафениба в качестве препарата первой линии в лечении светлоклеточного МПКР варьирует от 5,7 до 9,1 мес. [22, 42], общая выживаемость (при использовании последующих линий таргетной терапии) - от 27,1 до 33 мес. $[43,44]$.

Эффективность и безопасность применения сорафениба также подтверждена в 2-х открытых исследованиях с расширенными критериями включения ARCCS (ARCCS - Advanced Renal Cell Carcinoma Sorafenib), проводившихся в странах

\section{Сорафениб (Нексавар) относится к группе мультикиназных ингибиторов, действующих одновременно как на тирозинкиназы рещепторов, так и на серин/треониновые киназы в опухолевых и эндотелиальных клетках}

Северной Америки и Европы с участием 2504 и 1155 больных соответственно [45-47].

В открытом исследовании сорафениба с расширенными критериями включения ARCCS, проводившемся в США и Канаде, участвовали 2504 больных МПКР, которым невозможно было назначить таргетную терапию в рамках других исследований. В исследовании принимали участие в т. ч. пациенты с несветлоклеточными опухолями, метастазами в головной мозг, пожилого возраста, а также больные, ранее получавшие терапию другими препаратами. Согласно опубликованным результатам, полный ответ на лечение был зарегистрирован у 1 (0,05\%) из 1891 больного, частичный ответ - у 67 (4\%), стабилизация заболевания более 8 нед. у 1511 (80\%) пациентов, прогрессирование заболевания у 303 (16\%) больных. Суммарный ответ на лечение (сумма полных, частичных ремиссий и стабилизации) был достигнут у 84\% больных. Медиана выживаемости без прогрессирования составила 36 нед., а медиана общей выживаемости - 50 нед. Различий в эффективности терапии сорафенибом в зависимости от гистологического варианта ПКР, наличия метастазов в головной мозг, возраста и ранее проводившегося лечения отмечено не было. К наиболее часто встречающимся побочным эффектам II степени тяжести, связанным с приемом препарата, относились ладонно-подошвенный синдром (18\%), сыпь (14\%), гипертензия (12\%) и слабость $(11 \%)[45,47]$.

В открытом исследовании сорафениба с расширенными критериями включения, проводившемся в странах Европы (EU-ARCCS), приняли участие 1155 больных. В исследование включали в т. ч. пациентов с несветлоклеточными опухолями, бессимптомными метастазами в головной мозг, соматическим статусом по шкале ECOG 0-2 и ожидаемой продолжительностью жизни >2 мес. Медиана выживаемости без прогрессиро- вания составила 6,8 мес., а медиана продолжительности лечения 6,9 мес. Таким образом, эффективность и безопасность применения сорафениба оказались сопоставимы с проведенными ранее рандомизированными исследованиями [45].

Дополнительная активность сорафениба в качестве препарата второй линии лечения МПКР после неэффективности других таргетных препаратов была подтверждена результатами нескольких исследований. В ряде исследований с последовательным использованием сунитиниба и сорафениба подтверждено отсутствие перекрестной резистентности между препаратами, что позволяет использовать эти препараты последовательно [25-38].

В метаанализ, проведенный F. Stenner с соисследователями, включили 853 пациента из 13 исследований (11 ретро- и 2 проспективных). 398 пациентов получили последовательную терапию препаратами сунитиниб-сорафениб и 455 - сорафенибсунитиниб. Согласно полученным результатам, отмечено улучшение показателей суммарной выживаемости без прогрессирования в группе больных, получавших последовательность препаратов сорафениб-сунитиниб по сравнению с группой, получавшей сунитиниб-сорафениб: 15,4 против 12,1 мес. соответственно $(\mathrm{p}=0,0013)$. Статистически значимых различий во времени до первого прогрессирования выявлено не было вне зависимости от того, какой препарат был назначен в качестве терапии первой линии (медиана времени до первого прогрессирования на фоне сорафениба оказалась на 0,62 мес. больше по сравнению с сунитинибом, $\mathrm{p}=0,43)$. При назначении сорафениба после сунитиниба отмечено статистически значимое снижение выживаемости без прогрессирования по сравнению с назначением сунитиниба после сорафениба (среднее снижение на 2,66 мес., $p=0,003$ ). Таким образом, назначение сунитиниба в первой линии сопровождалось меньшей ВБП на фоне последующей терапии сорафенибом, в то время как терапия сорафенибом в первой линии не сопровождалась подобным снижением эффективности последующей терапии сунитинибом. Авторами был сделан вывод об отсутствии перекрестной резистентности между препаратами и целесообразности их последовательного применения. По мнению исследователей, последовательное применение схемы сорафениб-сунитиниб позволяет увеличить показатели выживаемости без прогрессирования по сравнению с последовательностью сунитинибсорафениб [37].

В настоящее время получены данные нескольких крупных рандомизированных исследований, в которых препарат сорафениб применяли как в первой, так и во второй линии терапии МПКР [19-24].

В открытое проспективное многоцентровое исследование III фазы (TIVO-1) по изучению эффективности препарата тивозаниб по сравнению с препаратом сорафениб у больных МПКР включили 517 больных, которых рандомизировали в соотношении 1:1. Согласно полученным данным, выживаемость без прогрессирования и частота обьективных ответов были статистически значимо лучше в группе больных, получавших тивозаниб, по сравнению с группой сорафениба; выживаемость без прогрессирования составила 11,9 мес. против 9,1 мес. ( $=0,042)$; частота обьективных ответов - 


\section{HEKCABAP ${ }^{\circ}$ \\ в лечении метастатического почечно-клеточного рака (ПКР)}

Новый подход . Эффективность. Надежность.

Опубликованы данные исследования

III фазы TARGET

Одобрен Европейским агентством по лекарствам (EMEA)

Первый препарат для перорального применения для лечения ПКР, одобренный Управлением по продуктам и лекарственным средствам США (FDA) 
$33 \%$ и 23\% ( $p=0,014)$ соответственно. При проведении подгруппового анализа выживаемости без прогрессирования у больных, ранее не получавших системной лекарственной терапии МПКР, медиана выживаемости без прогрессирования также была продолжительнее в группе тивозаниба, чем в группе сорафениба: 12,7 мес. против 9,1 мес. соответственно $(\mathrm{p}=0,037)$.

Однако при анализе общей выживаемости была выявлена тенденция к уменышению риска смерти на 20\% в группе сорафениба по сравнению с тивозанибом (отношение рисков $1,25, p=0,105$, медиана общей выживаемости в группе тивозаниба 28,8 мес., в группе сорафениба - 29,3 мес.). При этом 75\% больных в группе сорафениба получили терапию второй линии, в то время как в группе тивозаниба терапию второй линии получили лишь 36\% больных [22-24].

Отчасти это было связано с дизайном исследования, согласно которому после прогрессирования на фоне сорафениба больные имели возможность продолжить лечение тивозанибом, в то время как у больных из группы тивозаниба возможности продолжить лечение сорафенибом в рамках исследования не было.

\section{Сорафениб входит в перечень основных международных (NCCN, ESMO, EAU) и российских (Минздрава РФ) рекомендаций по лечению МПКР в качестве терапии первой и второй линии}

С другой стороны, в исследовании последовательной терапии эверолимусом и сунитинибом RECORD-3, в котором у пациентов также была возможность продолжать лечение вторым препаратом в рамках исследования после прогрессирования на фоне первого, лишь 52\% больных после сунитиниба и 54\% после эверолимуса смогли получить вторую линию терапии [48]. Поэтому нельзя исключить, что часть больных в исследовании TIVO-1 не получили последующую терапию после тивозаниба по причинам, не связанным с доступностью препаратов в рамках исследования.

В открытое многоцентровое рандомизированное исследование III фазы INTORSECT, посвященное сравнению эффективности и безопасности темсиролимуса и сорафениба у больных МПКР, у которых наблюдалось прогрессирование заболевания на терапии сунитинибом, включили 511 пациентов с МПКР. В исследование включили больных со статусом 0 или 1 по шкале ECOG. Медиана выживаемости без прогрессирования у больных, получавших темсиролимус, составила 4,28 мес. по сравнению с 3,91 мес. в группе больных, получивших сорафениб ( $p=0,1933)$. Однако при оценке общей выживаемости выявлены статистически значимые преимущества в группе больных, принимавших сорафениб, по сравнению с группой темсиролимуса, риск смерти в группе сорафениба был на 23,7\% меньше по сравнению с группой темсиролимуса, медиана общей выживаемости составила 16,64 мес. и 12,27 мес. соответственно (отношение рисков $1,31, \mathrm{p}=0,0144)$. Исследователи пришли к выводу, что темсиролимус не улучшает общую выживаемость в сравнении с сорафенибом во второй линии, и предположили, что применение ингибиторов VEGF, возможно, более целесообразно, чем ингибиторов mTOR у больных МПКР с прогрессированием заболевания после терапии сунитинибом [21].

На основании данных исследования TARGET препарат сорафениб одобрен в странах Европы (EMEA), США (FDA) и в 2007 г. зарегистрирован в РФ для лечения МПКР в первой и последующих линиях терапии. Сорафениб входит в перечень основных международных (NCCN, ESMO, EAU) и российских (Минздрава РФ) рекомендаций по лечению МПКР в качестве терапии первой и второй линии. Члены экспертных групп NCCN и ESMO по ПКР, основываясь на данных, полученных по окончании исследований II и III фазы и исследований сорафениба с расширенными критериями включения, рекомендуют применять сорафениб в качестве препарата первой линии терапии некоторым больным МПКР [9, 10, 49]. Рекомендуемые дозы для лечения сорафенибом - по 400 мг 2 раза/день перорально. Для удобства подбора дозировки при возникновении выраженных побочных эффектов препарат выпускается по 200 мг в 1 таблетке.

В настоящее время продолжаются исследования последовательной терапии при неэффективности или непереносимости других таргетных препаратов. Запланировано проведение ряда исследований III фазы по оценке эффективности и переносимости таргетных препаратов во второй линии лечения МПКР и исследования с последовательным назначением различных ингибиторов тирозинкиназ (табл. 2) [50].

Окончательная выработка четких рекомендаций по применению оптимальной последовательности таргетных препаратов станет возможной только после проведения крупных проспективных клинических исследований, а также после детального изучения механизмов резистентности. Поиск биологических характеристик опухоли и маркеров, варьирующих на фоне лечения, вероятно, поможет прогнозировать ответ на проводимое лечение.

\section{ЗАКЛЮЧЕНИЕ}

Прогноз течения заболевания больных МПКР с появлением таргетных препаратов значительно улучшился. В связи с развитием резистентности и непереносимостью препаратов на фоне длительной терапии в настоящее время в литературе активно обсуждаются вопросы разработки таргетных препаратов второй и третьей линии лечения МПКР с определением оптимальной последовательности их применения. Результаты большинства проведенных клинических исследований продемонстрировали целесообразность использования на первом этапе лечения препарата сорафениб с последующим назначением сунитиниба. Несомненно, для определения оптимальной последовательности использования таргетных препаратов необходимо завершение продолжающихся крупных проспективных клинических исследований III фазы.

Полный список литературы вы можете запросить в редакции. 